The Astrophysical Journal, 502:824-832, 1998 August 1

(C) 1998. The American Astronomical Society. All rights reserved. Printed in U.S.A.

\title{
DIAGNOSIS OF STELLAR WINDS AND TEMPERATURE STRUCTURES IN Be STARS THROUGH THE ANALYSIS OF Mg II LINES
}

\author{
LYdia S. Cidale ${ }^{1}$ \\ Facultad de Ciencias Astronómicas y Geofisicas, Paseo del Bosque S/N, 1900 La Plata, Buenos Aires, Argentina;lydia@fcaglp.fcaglp.unlp.edu.ar \\ Received 1997 June 30; accepted 1998 March 10
}

\begin{abstract}
We compute non-LTE Mg II line profiles for Be stars by considering 12 energy-level atoms and supposing that the circumstellar medium can be described by an expanding, spherically symmetric flow in which we assume the presence of a chromosphere. The line radiative transfer equation was solved rigorously in spherical coordinates and in the comoving frame. The $\mathrm{Mg}$ II line profiles predicted by this model coincide with those observed in some Be stars. The calculation was performed for a range of effective temperatures representative of the B spectral type. We have also analyzed the influence on the line profiles of different temperature and velocity distributions in the circumstellar material. The line spectrum variability of a $\mathrm{Be}$ star is interpreted as the result of a variable outward mass flux.
\end{abstract}

Subject headings: circumstellar matter — line: profiles — radiative transfer — stars: emission-line, $\mathrm{Be}-$ stars: mass loss

\section{INTRODUCTION}

Be stars exhibit a great variety of phenomena that have suggested the existence of an extended atmosphere, for example, rapid-rotation, emission lines in $\mathrm{H}$ and in singleionized elements, and IR and radio excesses. Optical interferometers in the $\mathrm{H} \alpha$ line (Quirrenbach 1994; Quirrenbach et al. 1997) reveal the existence of flattened envelopes around Be stars. The UV observations provide further detailed information about the properties of the stellar environment. These observations actually reveal details of (1) a cool circumstellar material, (2) a superionized region, and (3) high-velocity stellar winds. Be stars also display light and spectrum variability. The line spectrum variability is such that the same star, at different epochs, may undergo the B-normal, Be, and Be-shell phases; however, the temporal progression of this transition is very slow. Descriptions of the different events observed in Be stars are summarized by Doazan (see Underhill \& Doazan 1982).

We shall attempt to model the physical structure of the extended atmosphere of a Be star following the thermodynamical scheme proposed by Ringuelet \& Iglesias (1991), which, in a radial sequence, consists of (1) a quasi-thermal photosphere, (2) a chromosphere, where nonradiative and nonhydrostatic equilibrium develops and the temperature reaches a maximum, and (3) a cool region. The general characteristics of this model are described in $\S 2$. We suppose that the circumstellar envelope of the star can be represented, in a first approximation, by an expanding spherically symmetric medium.

Although a spherically symmetric medium is not the best geometrical representation for the extended envelope of $\mathrm{Be}$ stars, the spherical configuration (which is a zero-order model) allows us to distinguish and analyze the contribution of a stellar wind to line profiles, free of those effects that could arise because of the shape of the envelope. In fact, we have found that the velocity fields play the main role in the line formation process and that they could be responsible for the spectrum and light variabilities.

\footnotetext{
${ }^{1}$ Member of the Carrera del Investigador Científico, CONICET, Argentina.
}

The model that we have adopted (called " chromosphericwind model") was able to reproduce satisfactorily the $\mathrm{H} \alpha$ emission-line profile (Cidale \& Ringuelet 1993, hereafter CR), and it was also applied to interpret the IR continuum radiation excess of some Be stars (Vázquez, Cidale, \& Ringuelet 1993, hereafter VCR).

The calculation of the $\mathrm{H} \alpha$ line by $\mathrm{CR}$, applying monotonically increasing velocity laws, demonstrated that the main features in the profile depend upon the radial velocity gradients in the immediate subphotosphere and the base of the wind. Low-velocity gradients in the layers close to the photosphere yield a double-peaked emission, while highvelocity gradients in the same region yield P Cygni-type profiles. Since the mass-loss rate depends strongly on the radial velocity and its gradient at the photospheric radius, the chromospheric-wind model provides an alternative interpretation of the variability of the Be phenomena as the result of variable mass flow.

The aim of this paper is to continue studying the physical properties of the extended atmospheres of Be stars through the theoretical analysis of spectral lines. Of great interest for this purpose is the behavior of the $\mathrm{Mg}$ II resonance lines, because they are one of the most relevant features observed in the mid-ultraviolet stellar spectra around $2800 \AA$, and not much theoretical work has been done involving this feature.

In some Be stars, the $\mathrm{Mg}$ II $h$ and $k$ lines at $2800 \AA$ sometimes appear as pure absorptions and sometimes display emission wings and absorption cores (Dachs 1980; Doazan et al. 1991). The presence of Mg II emission seems not to be related to the spectral type and does not correlate with $v \sin i$ (Slettebak 1994). Some Be stars show blueshifted variable absorption components in the -50 to $-200 \mathrm{~km}$ $\mathrm{s}^{-1}$ range or exhibit P Cygni profiles (see Thomas 1983). In the photographic region, $\mathrm{Mg}$ II $\lambda 4481$ is typically seen in absorption down to spectral class B5 V; it persists up to B2 for luminosity class IV and may even be stronger in luminosity class III.

Previous calculations of the spectrum of $\mathrm{Mg}$ II were restricted to normal $\mathrm{B}$ and $\mathrm{O}$ stars; thus we can mention those performed by Lamers (1971), with an LTE solution, and by Mihalas (1972). Mihalas did work out a strictly 


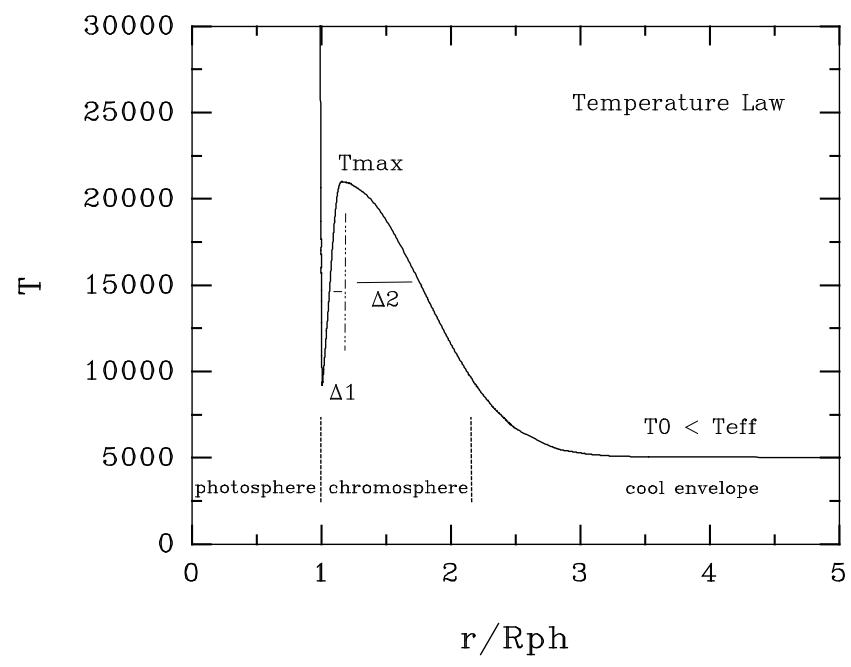

Fig. 1.-Temperature law used in the calculation of the models

consistent solution of the transfer and statistical equilibrium equations in a static plane-parallel medium; his computations provide a good fit to the observed equivalent widths of the line $\lambda 4481$, assuming a solar abundance for magnesium.

Calculations of non-LTE resonance line profiles of $\mathrm{Mg}$ II in early A-type stars, in an expanding atmosphere, were performed by Kunasz \& Praderie (1981) and by Catala, Kunasz, \& Praderie (1984). To study the Mg II lines, they adopted a two-level-atom approach, and the radiative transfer problem in the comoving frame was solved for a spherically symmetric wind. It appears that the doublet $\mathrm{Mg}$ II $\lambda 2800$ provides global information about the wind atmospheric structure, since these lines are formed over large regions.

In this paper, we discuss our results on the line spectrum of $\mathrm{Mg}$ II, which arises from a nonrotating and spherically expanding medium, by means of a rigorous treatment of the radiative transfer problem and by considering a non-LTE multilevel model atom. We investigate the effects on the line profiles that can be explained solely with a stellar wind by adopting different temperature and velocity laws for the extended atmosphere. Our discussion is based on a grid of models. Some of the models that we present were previously used in CR for the interpretation of the $\mathrm{H} \alpha$ line.

We find that the resonance lines permit an outlining of the velocity and temperature structure of the circumstellar medium. Therefore, an expanding envelope would be responsible for the presence of the blueshifted absorption components that have been reported by Thomas (1983) and by Morgan, Kondo, \& Modisette (1977).

\section{THE ATMOSPHERIC MODEL}

In order to take into account the different ionization stages and Doppler shifts observed in the line spectrum of Be stars, we adopt the chromospheric-wind model. Since the fundamental concepts and the details about the construction of the model were described in CR, VCR, and the references therein, we consider it appropriate to present here only the main characteristics of the model and to discuss its applicability.

We assume the existence of a sequence of spherically symmetric layers made up of a mixture of $\mathrm{H}$ and $\mathrm{He}$. The principal absorption sources for the continuous radiation are bound-free and free-free transitions of $\mathrm{H}_{\text {and }} \mathrm{H}^{-}$, Rayleigh scattering by $\mathrm{H} \mathrm{I}$ atoms, and Thomson scattering.

As we move outward through the extended atmosphere, we distinguish three regions with different thermodynamical and kinematical properties:

1. A classical photosphere in radiative and hydrostatic equilibrium with temperature and density distributions represented by a Kurucz (1979) model up to the photospheric radius, $R_{\mathrm{ph}}$ (defined as the radius, where $T=T_{\text {eff }}$ ). Above the radius $R_{\mathrm{ph}}$, the photospheric layers are still in radiative

TABLE 1

PARAMETERS OF THE MODELS

\begin{tabular}{|c|c|c|c|c|c|c|c|}
\hline Model & $\begin{array}{l}T_{\text {eff }} \\
(\mathrm{K})\end{array}$ & $\begin{array}{c}\Delta_{1} \\
\left(R_{*}\right)\end{array}$ & $\begin{array}{c}\Delta_{2} \\
\left(R_{*}\right)\end{array}$ & $\begin{array}{c}T_{0} \\
(\mathrm{~K})\end{array}$ & $\begin{array}{l}T_{\max } \\
(\mathrm{K})\end{array}$ & $\begin{array}{c}\text { Velocity } \\
\text { Law }\end{array}$ & $\begin{array}{c}\dot{M} \\
\left(M_{\odot} \mathrm{yr}^{-1}\right)\end{array}$ \\
\hline & 25000 & 1.5 & 1.5 & 16000 & 40000 & $\mathrm{~V}_{1}$ & $1.1 \times 10^{-7}$ \\
\hline $2 .$. & 25000 & 1.5 & 1.5 & 8000 & 40000 & $\mathrm{~V}_{1}$ & $1.1 \times 10^{-7}$ \\
\hline 3. & 25000 & 1.5 & 1.5 & 6000 & 40000 & $\mathrm{~V}_{1}$ & $1.1 \times 10^{-7}$ \\
\hline & 25000 & 1.5 & 1.5 & 5000 & 40000 & $\mathrm{~V}_{1}$ & $1.1 \times 10^{-7}$ \\
\hline & 25000 & 1.5 & 1.5 & 4500 & 40000 & $\mathrm{~V}_{1}$ & $1.1 \times 10^{-7}$ \\
\hline & 25000 & 1.5 & 1.5 & 4000 & 40000 & $\mathrm{~V}_{1}$ & $1.1 \times 10^{-7}$ \\
\hline $7 \ldots \ldots$ & 25000 & 1.5 & 1.5 & 6000 & 40000 & $\mathrm{~V}_{2}$ & $4.1 \times 10^{-9}$ \\
\hline $8 \ldots \ldots$ & 25000 & 0.08 & 1.5 & 8000 & 40000 & $\mathrm{~V}_{1}$ & $1.1 \times 10^{-7}$ \\
\hline $9 \ldots \ldots$ & 25000 & 1.5 & 1.5 & 8000 & 26000 & $\mathrm{~V}_{1}$ & $1.1 \times 10^{-7}$ \\
\hline 10. & 25000 & 0.08 & 1.5 & 8000 & 40000 & $\mathrm{~V}_{3}$ & $3.1 \times 10^{-9}$ \\
\hline $11 \ldots \ldots$ & 25000 & 1.5 & 1.5 & 8000 & 40000 & $\mathrm{~V}_{3}$ & $3.0 \times 10^{-9}$ \\
\hline $12 \ldots \ldots$ & 14000 & 0.08 & 1.5 & 6000 & 21000 & $\mathrm{~V}_{3}$ & $4.0 \times 10^{-10}$ \\
\hline $13 \ldots \ldots$ & 14000 & 1.5 & 1.5 & 6000 & 21000 & $\mathrm{~V}_{3}^{3}$ & $3.8 \times 10^{-10}$ \\
\hline $14 \ldots$ & 14000 & 2.0 & 1.5 & 5000 & 21000 & $\mathrm{~V}_{4}$ & $3.6 \times 10^{-10}$ \\
\hline $15 \ldots \ldots$ & 14000 & 2.0 & 1.5 & 5000 & 21000 & $\mathrm{~V}_{5}^{4}$ & $1.8 \times 10^{-9}$ \\
\hline $16 \ldots \ldots$ & 14000 & 2.0 & 1.5 & 5000 & 21000 & $\mathrm{~V}_{6}$ & $7.1 \times 10^{-9}$ \\
\hline $17 \ldots \ldots$ & 14000 & 2.0 & 1.5 & 5000 & 21000 & $\mathrm{~V}_{7}$ & $2.4 \times 10^{-8}$ \\
\hline $18 \ldots \ldots$ & 10000 & 0.08 & 1.5 & 6000 & 17000 & $V_{3}^{\prime}$ & $5.9 \times 10^{-11}$ \\
\hline $19 \ldots \ldots$ & 10000 & 1.5 & 1.5 & 6000 & 17000 & $\mathrm{~V}_{3}$ & $6.3 \times 10^{-11}$ \\
\hline $20 \ldots \ldots$ & 10000 & 0.08 & 1.5 & 6000 & 17000 & $\mathrm{~V}_{8}$ & $4.3 \times 10^{-9}$ \\
\hline
\end{tabular}

Note-The models were computed with a photospheric radius $R_{\mathrm{ph}}=8.81 \times 10^{11} \mathrm{~cm}$ for $T_{\text {eff }}=25,000 \mathrm{~K}, R_{\mathrm{ph}}=4.42 \times 10^{11} \mathrm{~cm}$ for $T_{\text {eff }}=14,000 \mathrm{~K}$, and $R_{\mathrm{ph}}=1.75 \times 10^{11} \mathrm{~cm}$ for $T_{\text {eff }}=10,000 \mathrm{~K}$. 
TABLE 2

VELOCITY LAWS

\begin{tabular}{|c|c|c|c|c|c|c|c|c|}
\hline$r / R_{\mathrm{ph}}$ & $\begin{array}{c}V_{1} \\
\left(\mathrm{~km} \mathrm{~s}^{-1}\right) \\
\end{array}$ & $\begin{array}{c}V_{2} \\
\left(\mathrm{~km} \mathrm{~s}^{-1}\right)\end{array}$ & 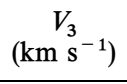 & $\begin{array}{c}\begin{array}{r}V_{4} \\
\left(\mathrm{~km} \mathrm{~s}^{-1}\right)\end{array} \\
\end{array}$ & $\begin{array}{c}V_{5} \\
\left(\mathrm{~km} \mathrm{~s}^{-1}\right)\end{array}$ & $\begin{array}{c}V_{6} \\
\left(\mathrm{~km} \mathrm{~s}^{-1}\right)\end{array}$ & $\begin{array}{c}V_{7} \\
\left(\mathrm{~km} \mathrm{~s}^{-1}\right)\end{array}$ & $\begin{array}{c}V_{8} \\
\left(\mathrm{~km} \mathrm{~s}^{-1}\right) \\
\end{array}$ \\
\hline $1 \ldots \ldots \ldots$ & 0.001 & 0.001 & 0.001 & 0.001 & 0.001 & 0.001 & 0.001 & 0.001 \\
\hline $1.01 \ldots \ldots$ & $\ldots$ & $\ldots$ & $\cdots$ & $\ldots$ & $\ldots$ & & 0.69 & 0.7 \\
\hline 1.05. & & 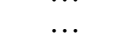 & $\ldots$ & $\ldots$ & ... & 1 & & \\
\hline $1.1 \ldots \ldots$ & 3.5 & $\ldots$ & & $\ldots$ & $\ldots$ & & 7 & 13 \\
\hline $1.2 \ldots \ldots$ & 20.2 & $\ldots$ & 0.2 & $\ldots$ & 1 & 4 & $\ldots$ & 55 \\
\hline $1.3 \ldots \ldots$ & $\ldots$ & & & $\ldots$ & $\ldots$ & $\ldots$ & 50 & 80 \\
\hline $1.5 \ldots \ldots$ & $\ldots$ & 0.7 & 0.35 & & $\ldots$ & & 70 & $\ldots$ \\
\hline $2 \ldots \ldots \ldots$ & $\ldots$ & & 20 & 1 & $\ldots$ & 20 & 90 & $\ldots$ \\
\hline $2.2 \ldots \ldots$ & & 4 & $\ldots$ & $\ldots$ & $\ldots$ & $\ldots$ & $\ldots$ & $\ldots$ \\
\hline $2.4 \ldots \ldots$ & 87.6 & $\ldots$ & $\ldots$ & $\ldots$ & $\ldots$ & $\ldots$ & $\ldots$ & $\ldots$ \\
\hline $2.6 \ldots \ldots$ & 101 & $\ldots$ & $\ldots$ & $\ldots$ & $\ldots$ & & & \\
\hline $3 \ldots \ldots \ldots$ & & 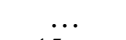 & $\ldots$ & 4 & 4 & 60 & 110 & 120 \\
\hline $3.3 \ldots \ldots$ & 112 & 15 & $\ldots$ & $\ldots$ & $\cdots$ & $\cdots$ & $\ldots$ & $\ldots$ \\
\hline 4.0 . & & 35 & $\ldots$ & $\ldots$ & $\ldots$ & $\ldots$ & $\ldots$ & $\ldots$ \\
\hline 4.9 . & 126 & & 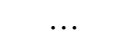 & & & 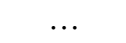 & $\ldots$ & \\
\hline 5. & & & ... & 20 & 20 & $\ldots$ & $\ldots$ & $\ldots$ \\
\hline 5.8 . & $\ldots$ & 98 & $\ldots$ & & & $\cdots$ & $\ldots$ & $\ldots$ \\
\hline $7 \ldots \ldots \ldots$ & $\ldots$ & 120 & & 60 & 60 & & $\ldots$ & \\
\hline $10 \ldots$ & $\ldots$ & $\cdots$ & 50 & 113 & 113 & 113 & $\ldots$ & 175 \\
\hline $20 .$. & $\ldots$ & $\ldots$ & & 120 & 120 & 120 & & \\
\hline $30 \ldots \ldots \ldots$ & & & & & & & 145 & $\ldots$ \\
\hline $36 \ldots \ldots \ldots$ & 132 & 132 & & $\cdots$ & $\cdots$ & $\cdots$ & $\cdots$ & $\ldots$ \\
\hline $40 \ldots \ldots \ldots$ & & & 120 & & & & & \\
\hline $50 \ldots \ldots \ldots$ & $\ldots$ & $\ldots$ & 260 & 120 & 120 & 120 & 150 & 230 \\
\hline $60 \ldots \ldots \ldots$ & . & & 295 & & & & & \\
\hline $70 \ldots \ldots .$. & 133 & 133 & 300 & 120 & 120 & 120 & 150 & 270 \\
\hline
\end{tabular}

Note-In the calculation the velocity laws are specified as a piecewise linear function of $r / R_{\mathrm{ph}}$.

equilibrium, but a soft expansion develops, and therefore, the mass density distribution results from the continuity equation.

2. An expanding high-temperature region (the chromosphere), where the temperature increases with radius from photospheric values up to a maximum value $\left(T_{\max }>T_{\text {eff }}\right)$ and then decreases to temperatures lower than $T_{\text {eff }}$. The velocity law, which increases monotonically with radius throughout the extended atmosphere until it reaches

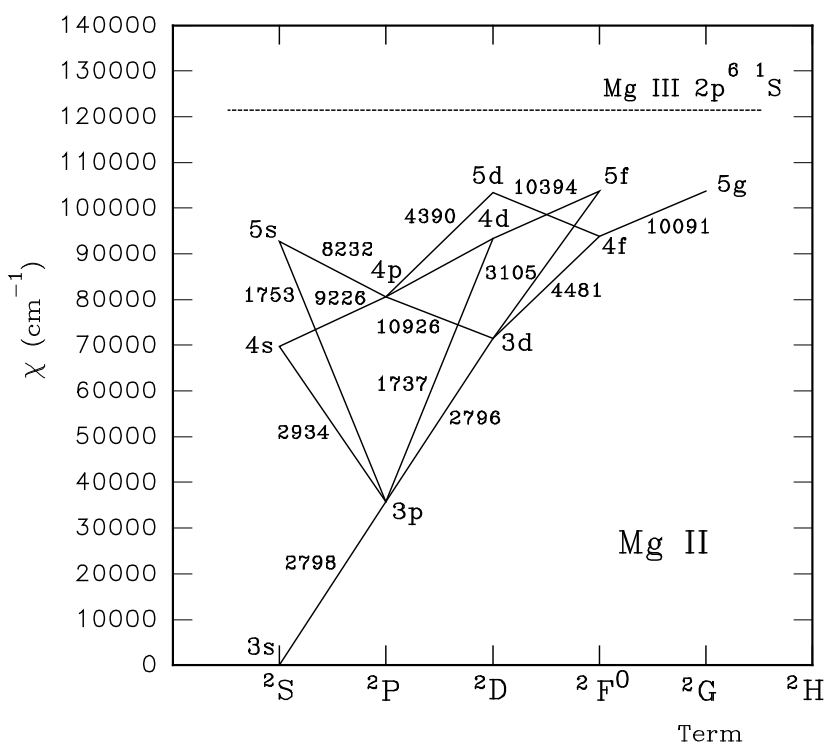

FIG. 2.-Schematic partial Grotrian diagram of the $\mathrm{Mg}$ II atom, showing the transitions considered explicitly in the self-consistent solution of transfer and statistical equilibrium equations. a constant value, is given in advance, and the mass density is computed through the continuity equation.

3. A cool envelope, which is isothermally expanding with $T=T_{0} \leq 10^{4} \mathrm{~K}$.

The temperature distribution, shown in Figure 1, is obtained from the analytical expression given in CR. The position of the maximum temperature $\left(R_{\mathrm{ch}}\right)$ and the chromospheric extension are controlled by two free parameters, $\Delta_{1}$ and $\Delta_{2}$, given in stellar radii. The value of the parameter $T_{\max }$ is selected in accordance with the results of Sutherland \& Dopita (1993), who considered collision, photoionization, and charge-exchange processes in the ionization balance of superionized elements.

Table 1 describes the most representative models, and Table 2 gives the prescribed velocity fields. We try different velocity curves with high-velocity and low-velocity gradients at the base of the chromosphere for a grid of model atmospheres with $10,000 \mathrm{~K} \leq T_{\text {eff }} \leq 25,000 \mathrm{~K}$ and $\log g=3.5$.

Taking into account the Mihalas (1972) results, we assumed a solar abundance for magnesium, relative to $\mathrm{H}$, $\alpha_{\mathrm{Mg}}=3.72 \times 10^{-5}$ (Böhm-Vitense 1965).

\section{THE ATOMIC MODEL}

For $\mathrm{Mg}$ II we used a model atom that is quite similar to the one adopted by Mihalas (1972) paper, where we find detailed information about the atomic parameters.

We included explicitly all levels up to $5 g$, while levels $6 p-6 h$ were treated as a single averaged state. This atomic configuration allows us to analyze mainly the resonance lines and the transition $\lambda 4481$, which connects levels $3 d$ and $4 f$. In this atomic model, $\mathrm{Mg}$ III is represented as a one-level atom. The adopted atomic configuration gives origin to 
such a large number of transitions that an explicit computational treatment of all these levels would be rather difficult. Therefore we selected a set of 15 lines for a self-consistent treatment. This selection was made by considering (1) the largest number of transitions connecting levels with the ground state, (2) all the transitions from levels $3 d$ and $4 f$, and (3) the strongest lines that connect levels $3 d$ and $4 f$ with the other levels. The atomic model used in the present calculation is shown in Figure 2.

Transition probabilities were taken from Wiese, Smith, \& Miles (1969), when available; otherwise, Coulombapproximation or hydrogenic values were used. The values used in each case correspond to the entire multiplet averaged over all components.

We adopted the photoionization and collisional cross sections that were compiled by Mihalas (1972).

Since the $\mathrm{Mg}$ I lines are generally weak or absent in the spectrum of a Be star, we did not take into account any explicit transitions with these atoms in the calculation. We assumed that $\mathrm{Mg} \mathrm{I}$ is in ionization equilibrium with the fundamental stage of $\mathrm{Mg}$ II.

\section{THE COMPUTATIONAL PROCEDURE}

We have dealt with a self-consistent solution of the radiative transfer problem in the comoving frame, for expanding flows with spherical symmetry, and the statistical equilibrium equations for multilevel atoms. Basically, we have used the method proposed by Mihalas \& Kunasz (1978), and the statistical equilibrium equations are solved for a $\mathrm{Mg}$ II atomic model consisting of 12 energy levels.

The calculation was initiated by solving the rate equations on the assumption of detailed radiative balance in all lines. The resulting populations were used to generate source and sink terms to solve the transfer equations in all lines by means of the equivalent two-level-atom approach. The new computed radiation field was used to revise the populations by solving the statistical equilibrium equations. This procedure is continued until reaching the convergence of the populations. The lines are assumed to be formed with complete redistribution over the profile function, which is considered to be a Gaussian profile.

\section{RESULTS AND DISCUSSION}

Once the atmospheric model and the velocity field have been selected, the line radiative transfer equation was solved for the $\mathrm{Mg}$ II atoms, as mentioned in the preceding sections.

We are not modeling any particular star. We only want to present general results that allow us to obtain direct relations between the computed line features and the physical parameters of the atmosphere, such as temperatures, terminal velocities, mass loss, and so on.

We computed $\mathrm{Mg}$ II lines for a wide range of effective temperatures. To present these results, three ranges of spectral types were selected: the early types, with an effective temperature between 25,000 and $20,000 \mathrm{~K}$; the middle types, around B5 or B6, with $T_{\text {eff }}=14,000 \mathrm{~K}$; and the late types, B9 or A0, with $T_{\text {eff }}=10,000 \mathrm{~K}$. We find that the profiles of the resonance lines are very sensitive to the temperature structure and to the velocity and mass-density distributions.

\subsection{Influence of a Velocity Field upon the Resonance Lines}

We consider it appropriate to discuss first the role of the velocity field, maintaining the temperature configuration for the circumstellar medium fixed, since the shape of the profile is mainly determined by the velocity distribution.

Figure $3 a$ illustrates a $\mathrm{Mg}$ II resonance line ( 22802.7$)$ computed with two different velocity laws for a star with $T_{\text {eff }}=$ $25,000 \mathrm{~K}$. The ordinate axis is given in a velocity scale, and the corresponding velocity laws are plotted in Figure $3 b$. The velocity curve that is steeper in the region close to the photosphere yields a double absorption profile (Table 1, model 3), and the curve that is smoother yields a pure absorption profile (model 7).

The single symmetric absorption profile obtained with model 7 arises from the photosphere and the immediate upper layers where there is almost no expansion. The outer

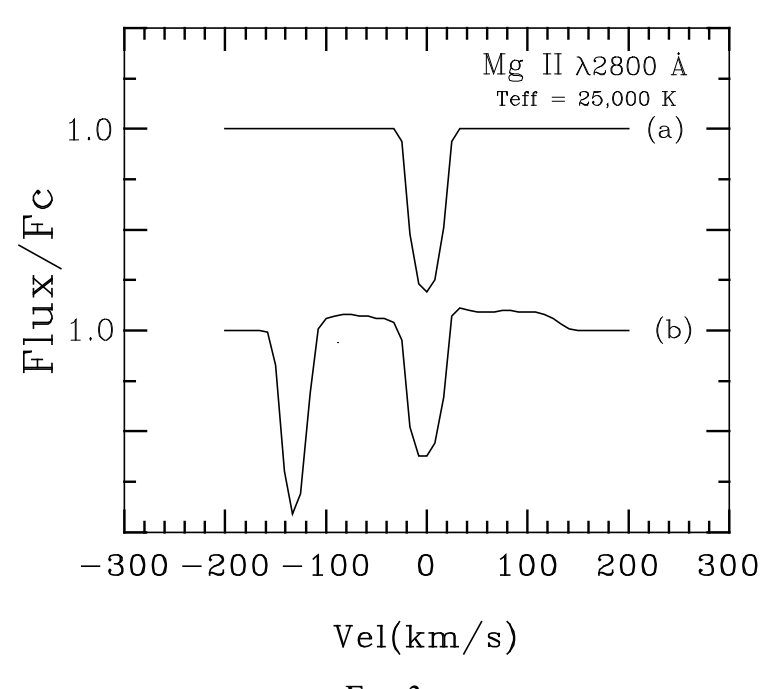

FIG. $3 a$

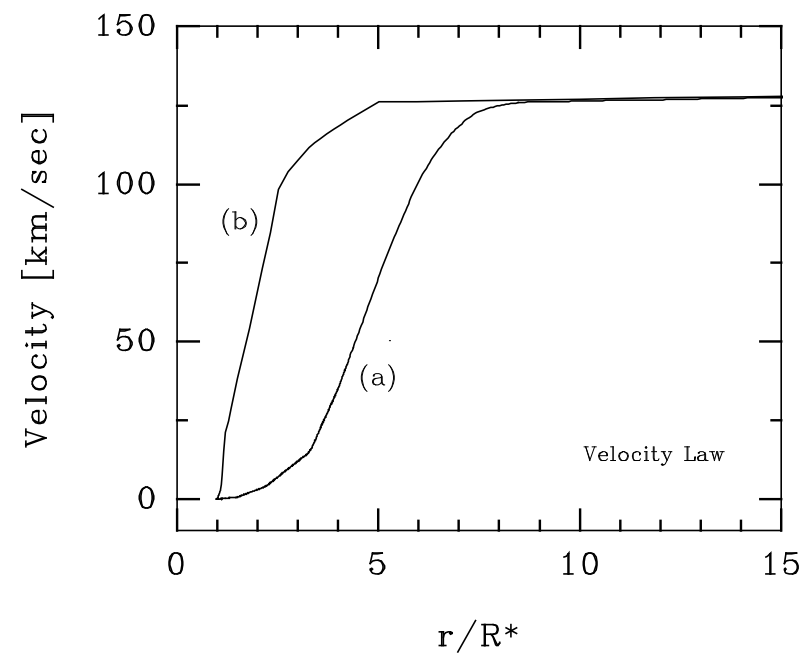

FIG. $3 b$

FIG. 3.-(a) Theoretical emergent line profiles of $\mathrm{Mg}$ II obtained with the velocity laws plotted in (b). Low-velocity gradients in the outer photospheric regions yield a single absorption line, curve (a), while high-velocity gradients in the same layers yield emissions and discrete absorption components, curve (b). (b) Two different velocity laws used to compute the $\mathrm{Mg}$ II resonance lines in (a). 
radius of the resonance-line-forming region $\left(R_{\max }\right)$ is less than 1.5 stellar radii. These layers can be considered in a quasi-hydrostatic equilibrium, and therefore the resulting profile shows similar features to those observed in a normal B star.

Model 3 corresponds to a higher mass-loss rate than model 7, and this implies a more extended line-forming region $\left(R_{\max }<63 R_{*}\right)$. We find that the profile with two absorption cores (model 3 ) is due to the contribution of two quite different regions; the undisplaced component originates mainly in the photospheric regions, as in model 7, while the blueshifted absorption and the emissions in the wings of the undisplaced component originate in the cool envelope.

Velocity curves with even smoother gradients than those of curve (a) yield a pure single absorption. Any other curve with velocity gradients between curves (a) and (b) predict profiles with two absorption cores but different intensity in the blueshifted component.

In Be stars of later spectral type, the behavior of the resonance lines with the velocity field is qualitatively similar to the cases of earlier spectral types. Thus, in Figure 4, we illustrate the line profiles as computed for a star with $T_{\text {eff }}=$ $14,000 \mathrm{~K}$ and different velocity laws (models 14,15 , and 16 ). In these particular cases, we have used the velocity distributions that had been considered in CR for the calculation of the $\mathrm{H} \alpha$ line.

The pure absorption line is obtained again with the lowest velocity gradient above the photospheric layers (model 14). As the velocity gradient increases in the neighborhood of the photosphere, more complex emission and/or absorption features result. For instance, the presence of a weak discrete absorption component, at $-120 \mathrm{~km} \mathrm{~s}^{-1}$, in the profile of the $\mathrm{Mg}$ II line (model 15) is obtained with a gradient of velocity that is slightly higher than the one of model 14. Even higher velocity gradients yield discrete absorption features as well as the emission that borders the low-velocity components (model 16).

Figure 5 shows a typical P Cygni profile that corresponds to winds with the highest acceleration above the photosphere (models 17 and 20). Models with higher mass-loss rate yield larger line-forming regions.

We conclude that the undisplaced absorption component originates in the photosphere, while the emission features and the displaced absorption component arise in the outer regions. In all cases, the core of the blueshifted absorption component reveals the velocities of the most external lineforming layers.

\subsection{Influence of the Temperature Structure upon the Resonance Lines}

In this section we will discuss the influence of the circumstellar temperature structure on the emergent line profile, since the $\mathrm{Mg}$ II resonance lines are collision dominated (Thomas 1957). For this discussion, we have to take into account the forming regions of the different components.

Let us consider the line components that originate in the cool extended envelope. Figure 6 exhibits a sequence of theoretical line profiles of the $\mathrm{Mg}$ II $3 s-3 p$ resonance transition at $\lambda \lambda 2795.5,2802.7$ computed with different values of $T_{0}$ (electronic temperature of the cool envelope) and the same velocity law for a star with $T_{\text {eff }}=25,000 \mathrm{~K}$ (Table 1 , models $1-6)$. Changes in $T_{0}$ yield intensity variations in the blueshifted absorption and in the wings of the undisplaced absorption component, which may appear in emission. The displaced absorption and the emissions display their maximum intensity at $6000 \mathrm{~K}$ (model 3), and both features disappear when the temperature of the outer envelope decreases to values lower than $4500 \mathrm{~K}$ (model 6).

The intensity of the displaced component also depends on the location of the high-temperature region and on its maximum temperature value (Fig. 7). It becomes deeper
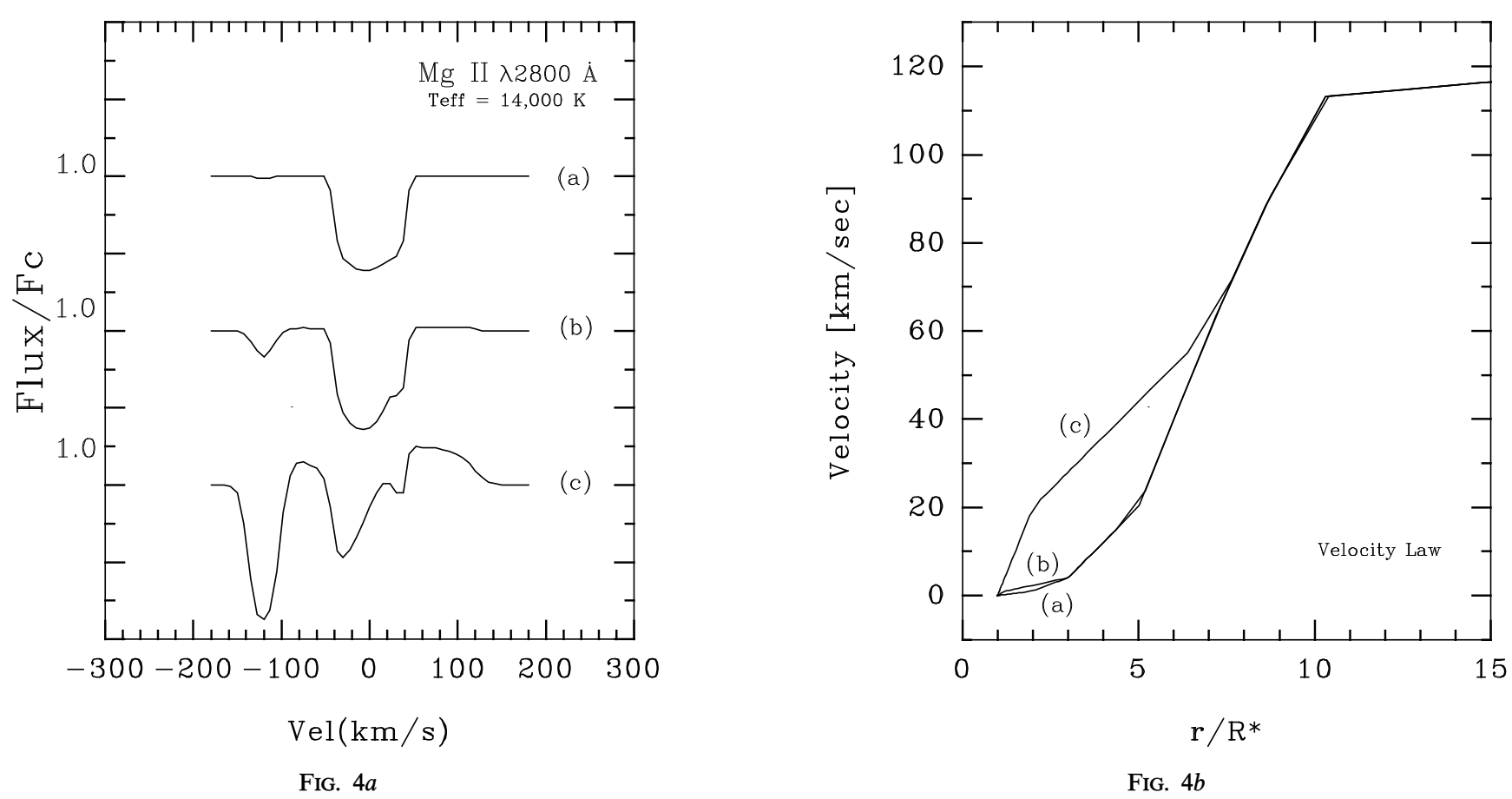

FIG. $4 b$

FIG. 4. - (a) Sequence of line profiles obtained with the velocity laws illustrated in $(b)$. The blueshifted component becomes deeper when increasing the velocity gradient at the base of the wind. (b) Velocity laws used in the calculation of the $\mathrm{Mg}$ II lines in $(a)$. 

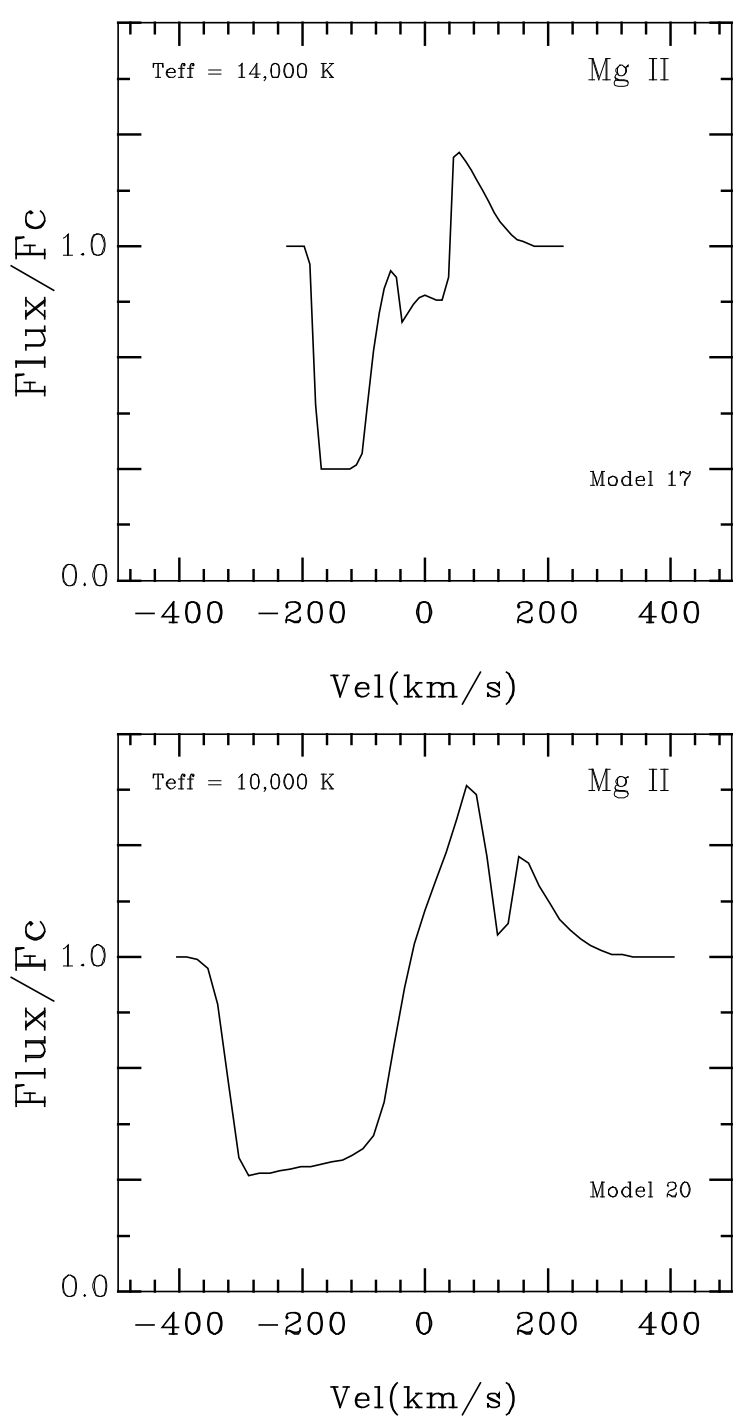

FIG. 5.-Typical P Cygni profiles computed for a $T_{\text {eff }}=14,000 \mathrm{~K}$ (model 17) and for $T_{\text {eff }}=10,000 \mathrm{~K}(\operatorname{model} 20)$.

when the chromosphere is located near the photospheric layers $\left(\Delta_{1}<1\right)$ and/or the $T_{\max }$ value decreases. The chromospheric extension, controlled by parameter $\Delta_{2}$, has negligible effects on the profile's global intensity. A small enhancement of the emission components does occur when this parameter increases. Both results confirm that the blueshifted absorption is mainly due to the contribution of the cool envelope.

We should emphasize that the response to the temperature of a line component emerging in the extended envelope is similar along the spectral range selected here. In contrast, the behavior of a photospheric line component is different for each spectral type, as will be shown later.

To analyze the peculiar behavior of the photospheric line component along the spectral range, we study the influence of the chromosphere upon the profiles by varying the parameter $\Delta_{1}$. In particular, to illustrate this result, we chose a low-gradient velocity law, since it yields a single profile.

Figure 8 presents, for each spectral type, $\mathrm{Mg}$ II line profiles obtained with the same velocity law but two different locations of the chromospheric region (a deep chromo- sphere and a far-out chromosphere, $\Delta_{1} \sim 0.1$ and $\Delta_{1}>1$, respectively).

Although we are using the chromospheric-wind model, we note that, in an early spectral type, there is no direct evidence of the existence of a high-temperature region, such as the presence of emission-line features. All the models with high $T_{\text {eff }}$ correspond to a line source function that decreases monotonically with radius. Therefore, the resulting line component that emerges in the photosphere and in the immediate postphotospheric regions is in absorption (Fig. 8 , models 10 and 11). Nevertheless, the photospheric line component is of a smaller equivalent width when the location of the chromospheric maximum temperature is located closer to the central star.

In middle spectral types, our model may show evidences of a deep chromosphere in the $\mathrm{Mg}$ II photospheric line component. In Figure 8, model 12, it is possible to observe the appearance of an emission structure inside the absorption profile. Its line source function starts to increase with increasing radii above the photospheric region (corresponding to the run of the temperature distribution), but immediately after and before the temperature reaches its maximum value it decreases with the radius. Again, the line equivalent width goes down if the position of the maximum temperature region is close to the photosphere.

The presence of the chromosphere can be highlighted in the resonance lines of a late Be star. The theoretical $\mathrm{Mg}$ II lines display prominent emissions when a deep chromospheric structure is considered (Fig. 8, model 18).

In relation to the chromospheric structure, we can say that it has a rather low influence upon the $\mathrm{Mg}$ II resonance lines in Be stars with $T_{\text {eff }} \geq 12,000 \mathrm{~K}$.

\subsection{The Subordinate Lines}

In all the cases we investigated, the $3 p-4 d$ transitions, multiplet UV 3 , and the transitions at $4481 \AA$ appear in absorption, and they do not vary under any variations of temperature and velocity structure. This implies that the lines originate mainly in the photospheric layers. Therefore, we think that the presence of emission features on these line profiles could be revealing a photosphere in neither radiative nor hydrostatic equilibrium. This kind of model needs to be further investigated.

\section{CONCLUSIONS}

With the chromospheric-wind model, we investigate the line formation processes of $\mathrm{Mg}$ II in nonisothermal media with both low-velocity and large-velocity gradients by means of a rigorous treatment of the line radiative transfer.

Since the atomic configuration of $\mathrm{Mg}$ II permits that some lines be collision dominated, the line source function would be coupled to the temperature structure of the atmospheric region where these lines arise. Thus, the $\mathrm{Mg}$ II lines may be used for the diagnostics of the thermodynamical structure of the extended envelope. The resonance lines also allow us to outline the radial velocity pattern of the circumstellar medium, since the shape of the profiles depends on the velocity field.

The results we have obtained with the chromosphericwind model can be summarized as follows:

1. The velocity law plays the main role in the line formation process; it defines the shape of the profile and the extension of the line-forming regions. 

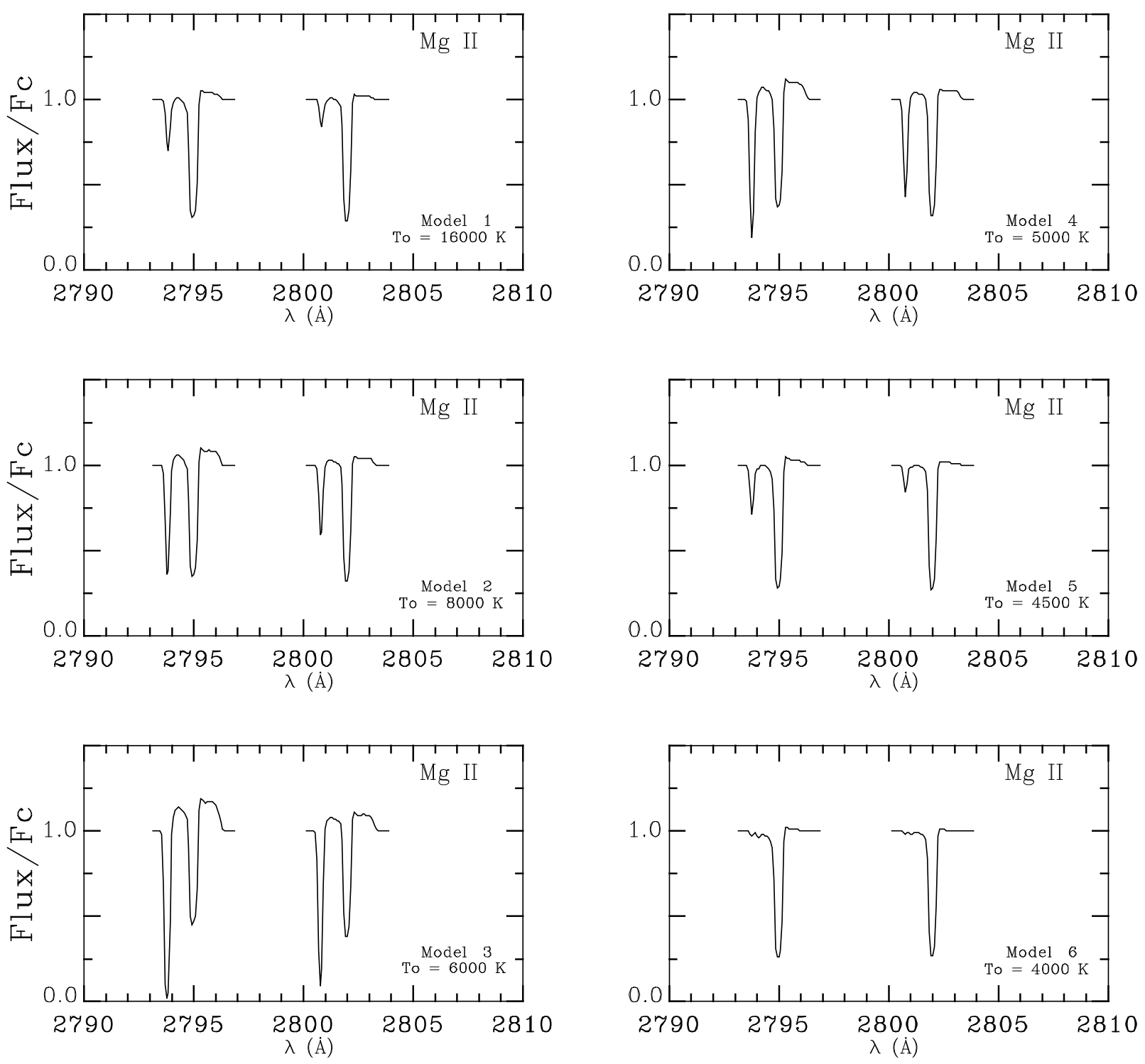

FIG. 6.-Intensity variations in the blueshifted and emission components due to the different temperature values adopted for the circumstellar envelope

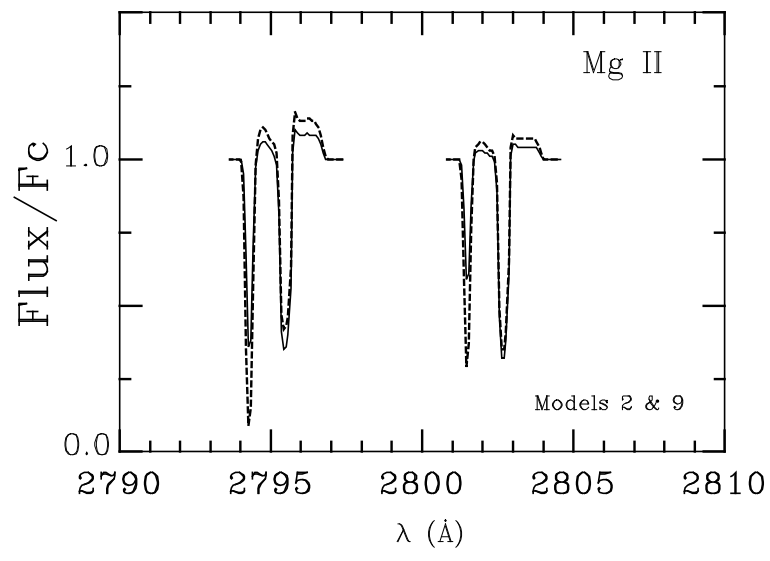

FIG. 7.- Mg II line profiles computed for different maximum temperature values, $T_{\max }=40,000$ (solid line) and $T_{\max }=26,000$ (dashed line). The absorption component is deeper when the temperature decreases.
2. Low gradients of velocity in regions close to the photosphere yield $\mathrm{Mg}$ II resonance lines with single absorption profiles that originate in the outer layers of the photosphere. Instead, higher velocity gradients in the same regions yield extended forming regions for resonance lines with double absorption components or P Cygni profiles. In the latter case, the core of the blueshifted component gives the velocity of the outer line-forming regions, which are generally located in the cool envelope.

3. The blueshifted narrow absorption is sensitive to the electronic temperature of the cool envelope and presents its maximum intensity around $6000 \mathrm{~K}$.

4. In spectral types with $T_{\text {eff }} \geq 12,000 \mathrm{~K}$, there is no direct evidence of line formation in the chromosphere, such as a line emission feature. The presence of a chromosphere fills the line with emission, yielding as the result absorption lines with lower equivalent widths. Instead, in a late B star the UV Mg II lines may appear in emission, when a deep 

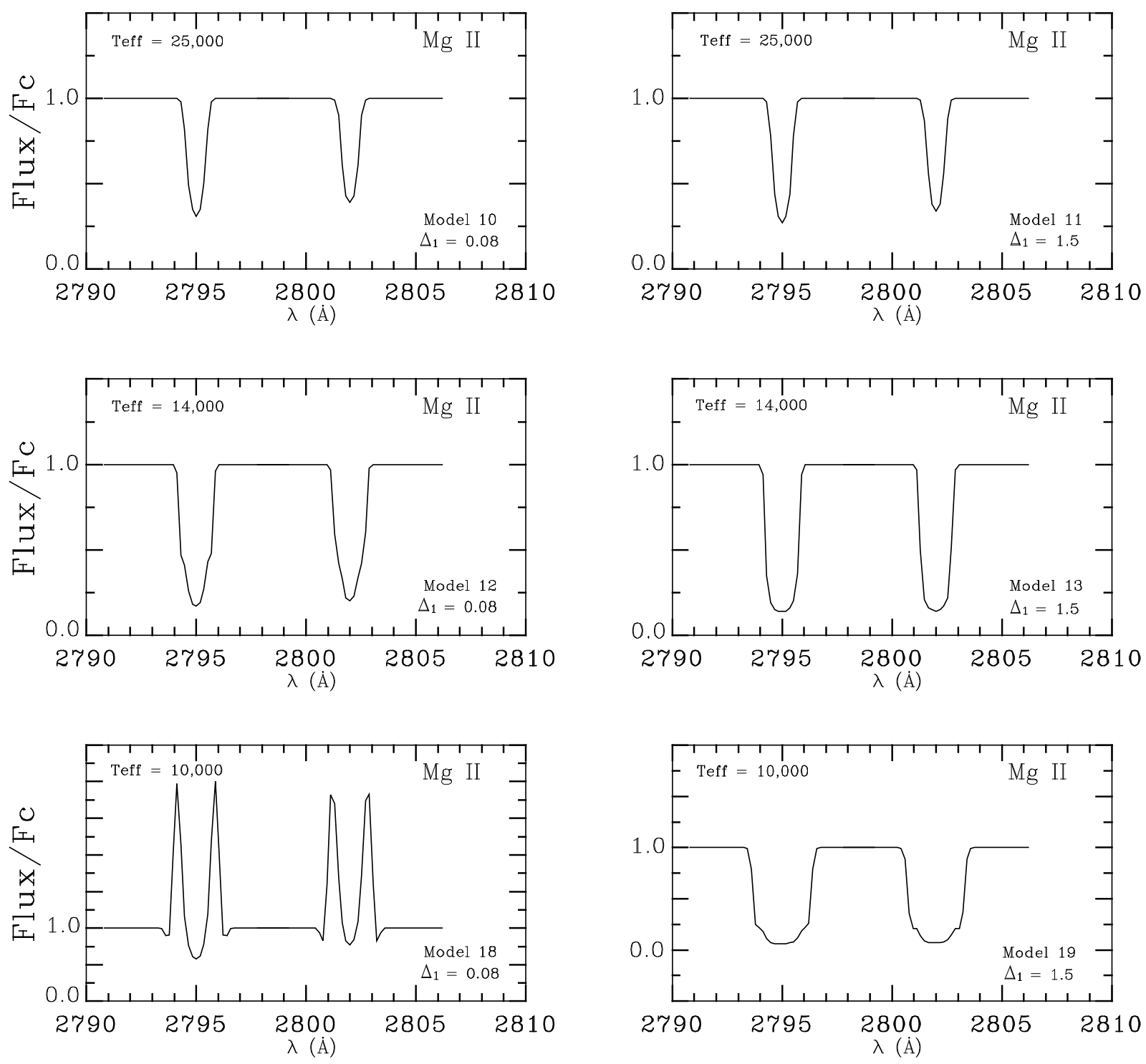

Fig. 8.-Influence of the chromosphere's location upon a photospheric line component in different spectral types

chromosphere model $\left(\Delta_{1} \sim 0.1\right)$ is considered. In this case, these lines originate in the chromosphere.

5. In all the models we have considered, the subordinated lines are restricted to the photospheric regions, and $\lambda 4481$ is always in absorption.

We have to keep in mind that the results presented in this paper were computed for a spherically symmetric medium with monotonically increasing velocity laws. We obtain all sorts of profiles, depending on the selected velocity and temperature distribution. Since we are dealing with an ad hoc model, a comparison of the computed profiles with the observed ones will place constraints on the parameters of the model.

The Mg II profiles computed with the chromosphericwind model present qualitatively similar features to those observed in some Be stars; a pure absorption profile, which is reproduced with a smooth expansion model, is the most common feature observed in a Be spectrum. Blueshifted discrete absorptions or P Cygni profiles are seen as well (Thomas 1983; Doazan et al. 1991); in particular, variable Be stars do display them. The stars $\gamma$ Cassiopeae and $\zeta$ Tauri have shown narrow components to each resonance doublet line shifted $-215 \mathrm{~km} \mathrm{~s}^{-1}$ and $-75 \mathrm{~km} \mathrm{~s}^{-1}$, respectively (Morgan et al. 1977).

From the IUE archives, we find that there are more $\mathrm{Be}$ stars that probably display a weak narrow component shifted to the blue: HD 23862, 87543, 110335, and 153261. It would be interesting to analyze their spectra in detail in order to study the properties of their stellar winds. P Cygni profiles, with characteristics that are quite similar to the ones we may compute, were observed in HD 50123, 50138, 94878, 94910, 19007, and 259431. Mg II discrete, narrow components were also identified in the spectrum of B supergiant stars (Bates \& Gilheany 1990).

We found that the shortward-shifted absorption components in the resonance transitions reveal the physical conditions of the outer regions and give information about the terminal velocity of the wind.

We want to remark that single absorption profiles with emission in the wings can be reproduced if we consider late Be stars with a chromospheric structure. Instead, we have not been able to reproduce these profiles in early Be stars; the computed profiles are in absorption, or they display emission in the wings when a strong blueshifted absorption 
component is present. This result could be due to the fact that we are considering velocity laws that increase monotonically with the radius, but $\mathrm{Mg}$ II emission presumably arises in a cool, low-velocity envelope (Lamers \& Snijders 1975). Since the profiles are very sensitive to the velocity fields, calculations with rotation and/or accelerating/ decelerating winds need to be explored. A decelerating wind yields denser outer regions, which could enhance the emission. But unfortunately, a rigorous computation of the radiative transfer equation for a rotating axisymmetric stellar wind is still technically impossible. Traditionally, this kind of research was done with the Sobolev approximation. Mazzali (1990) have shown that the introduction of rotation in the motion of stellar wind particles leads to much smoother P Cygni-type profiles than those obtained for pure expansion and may cause the forming of a blue-wing emission peak.
Our model predicts that in B stars with smooth expansion, the subordinated and resonance lines (that display a single absorption profile) are mainly formed in the photosphere. Therefore, in the scheme of our model, the variations observed in the absorption lines of some Be stars may be interpreted in terms of nonradial pulsation or photospheric oscillations theory, and the presence of any emission features in the subordinated lines would evidence large departures of radiative and hydrostatic equilibrium in the outer photospheric regions, since a temperature rise in the region would be necessary to explain these emissions. These particular cases would need to be explored with a photospheric dynamical model.

I would like to express my gratitude to A. Ringuelet for suggesting this research and for her critical comments on the manuscript.
Bates, B., \& Gilheany, S. 1990, MNRAS, 243, 320

Böhm-Vitense, E. 1965, in Landolt-Börnstein, Gruppe 6: AstronomieAstrphysik und Weltrumforschung, Band 1, ed. H. H. Voigt (Berlin: Springer), 398

Catala, C., Kunasz, P. B., \& Praderie, F. 1984, A\&A, 134, 402

Cidale, L. S., \& Ringuelet, A. E. 1993, ApJ, 411, 874 (CR)

Dachs, J. 1980, ESA SP-157, 139

Doazan, V., Sedmak, G., Barylak, M., \& Rusconi, L. 1991, A Be Star Atlas of Far UV and Optical High-Resolution Spectra (ESA SP-1147; Paris: ESA Sci. Publ.)

Kunasz, P. B., \& Praderie, F. 1981, ApJ, 247, 949

Kurucz, R. L. 1979, ApJS, 40, 1

Lamers, H. J. G. L. M. \& Snijders, M. A. 1975, A\&A, 41, 259

Mazzali, P. A. 1990, A\&A, 238, 191

Mihalas, D. 1972, ApJ, 177, 115

Mihalas, D. \& Kunasz, P. B. 1978, ApJ, 219, 635

Morgan, T. H., Kondo, Y., \& Modisette, J. L. 1977, ApJ, 216, 457

\section{REFERENCES}

Quirrenbach, A. 1994, IAU Symp. 162, Pulsation, Rotation, and Mass Loss in Early-Type Stars, ed. L. A. Balona, H. F. Henrichs, \& J. M. Le Contel (Dordrecht: Kluwer), 450

Quirrenbach, A., et al. 1997, ApJ, 479, 477

Ringuelet, A. E., \& Iglesias, M. E. 1991, ApJ, 369, 463

Slettebak, A. 1994, ApJS, 94, 163

Sutherland, R. S., \& Dopita, M. A. 1993, ApJS, 88, 253

Thomas, R. N. 1957, ApJ, 125, 260 240

Underhill, A., \& Doazan, V. 1982, in Be Stars with and without Emission Lines (NASA SP-456)

Vázquez, A. C., Cidale, L. S., \& Ringuelet, A. E. 1993, ApJ, 419, 286 (VCR)

Wiese, W. L., Smith, M. W., \& Miles, B. M. 1969, in Atomic Transition Probabilities, Vol. 2 (Washington, DC: US Dept. of Commerce, NBS), 29 